\title{
ON THE SUM OF CONTINUOUS AND DARBOUX FUNCTIONS
}

\author{
J. SMITAL
}

\begin{abstract}
The sum of a continuous function and a measurable function which maps each interval onto the reals can have a countable range. This is a solution of a problem of Bruckner and Ceder [1].
\end{abstract}

Bruckner and Ceder [1] have shown that the sum $f+g$, where $f: R \rightarrow R$ is absolutely continuous on some interval $I \subset R$, and $g$ is measurable and takes on each real value on each interval, must have uncountable range. They asked whether the theorem remains valid with absolute continuity replaced by continuity. The following theorem shows that the answer is "no".

THEOREM. There is a continuous function $f: R \rightarrow R$ of bounded variation, and a measurable Darboux function $g$ which takes each interval onto $R$, such that $f+g$ takes on only rational values.

REMARK. For each continuous $f$, which is not constant on any interval there is always some Darboux $g$ such that $f+g$ has countable range; however, if $f$ is absolutely continuous then $g$ must be nonmeasurable; see [1].

Proof of Theorem. To each open interval $I=(a, b) \subset[0,1]$ assign a set $D_{I} \subset I$ and a function $f_{I}:[0,1] \rightarrow[0,1]$ as follows: Fix some $c \in(a, b)$ and let $D_{1}$ (or $\left.D_{2}\right)$ be a nonempty perfect zero measure subset of $(a, c]$ (or of [ $c$, $b$ ), respectively). Put $D_{I}=D_{1} \cup D_{2}$. Let $h_{i}, i=1$, 2, be a nondecreasing continuous function $[0,1] \rightarrow[0,1]$ with $h_{i}\left(D_{i}\right)=[0,1]$. Put $f_{I}(x)=h_{1}(x)$ for $x \in[0, c]$, and $f_{I}(x)=1-h_{2}(x)$ otherwise.

Let $P_{1}=D_{(0,1)}$, and let $f_{1}=f_{(0,1)}$. Assume, by induction, that sets $\left\{P_{k}\right\}_{k<n}$ and functions $\left\{f_{k}\right\}_{k<n}$ have been constructed such that each $P_{k}$ is a nonempty perfect zero measure set. If $I$ is a component interval of $(0,1) \backslash \cup_{k<n} P_{k}$ with maximal length let $P_{n}=D_{I}, f_{n}=2^{-n} f_{I}$. Put $f^{*}=\Sigma_{n} f_{n}$. Clearly $f^{*}$ is continuous and has bounded variation since $V\left(f^{*}\right) \leqslant \Sigma_{n} V\left(f_{n}\right)$ $=3$.

Now we shall define a function $g^{*}:[0,1] \rightarrow R$. Let $\left\{r_{k}\right\}_{k=1}^{\infty}$ be a sequence of the rationals, and let $\left\{Q_{k}\right\}_{k=1}^{\infty}$ be a sequence of pairwise disjoint subsets of the set $Q$ of rationals such that each $Q_{k}$ is dense in $R$. Finally, let $A$ be a set, which for each real $u$ contains exactly one number from the set $u+Q$. For each natural $n$, and each $x \in A$, let $B_{n}(x)=\left(f^{*}\right)^{-1}\left(x+Q_{n}\right)$. Put $g^{*}(t)=$

Received by the editors February 3. 1976.

AMS (MOS) subject classifications (1970). Primary 26A15.

Key words and phrases. Darboux functions. 
$-x+r_{n}$ if $t \in P_{m} \cap B_{n}(x)$ for some $m, n, x$, and let $g^{*}(t)=-f^{*}(t)$ otherwise. The function $g^{*}$ is well defined since $B_{k}(x) \cap B_{l}(y)=\varnothing$ whenever $x \neq y$ or $k \neq l$.

For each $m, g^{*}\left(P_{m}\right)=R$. Indeed, let $z \in R$. Put $\{y\}=(-z+Q) \cap A$, and choose natural $s$ such that $y=-z+r_{s}$. It is easy to verify that $P_{m} \cap B_{s}(y) \neq \varnothing$ and that for $t \in P_{m} \cap B_{s}(y)$ we have $g^{*}(t)=-y+r_{s}=$ $z$.

Now since each open subinterval $I$ of $[0,1]$ contains some $P_{m}$ there is $g^{*}(I)=R$. Since $\cup_{k} P_{k}$ has zero measure, and $g^{*}(t)=-f^{*}(t)$ for $t \notin$ $\cup_{k} P_{k}, g^{*}$ is measurable.

To see that the range of $f^{*}+g^{*}$ is contained in $Q$ let $t \in P_{m} \cap B_{n}(x)$ for some $m, n, x$. Then there is $r \in Q_{n}$ such that $f^{*}(t)=x+r$ and

$$
f^{*}(t)+g^{*}(t)=f^{*}(t)-x+r_{n}=r+r_{n} \in Q \text {. }
$$

In the case $t \notin \cup_{m} \cup{ }_{n} \cup{ }_{x}\left(P_{m} \cap B_{n}(x)\right)$, clearly $f^{*}(t)+g^{*}(t)=0$.

To finish the proof it suffices to define $f(x)=2^{-|n|} f^{*}(x-n)$ and $g(x)=$ $2^{-|n|} g^{*}(x-n)$ whenever $x \in[n, n+1)$, for each nonzero integer $n$.

\section{REFERENCES}

1. A. M. Bruckner and J. Ceder, On the sum of Darboux functions, Proc. Amer. Math. Soc. 51 (1975), 97-102.

Department of Mathematics, Komensky University, 81631 Bratislava, Czechoslovakia 